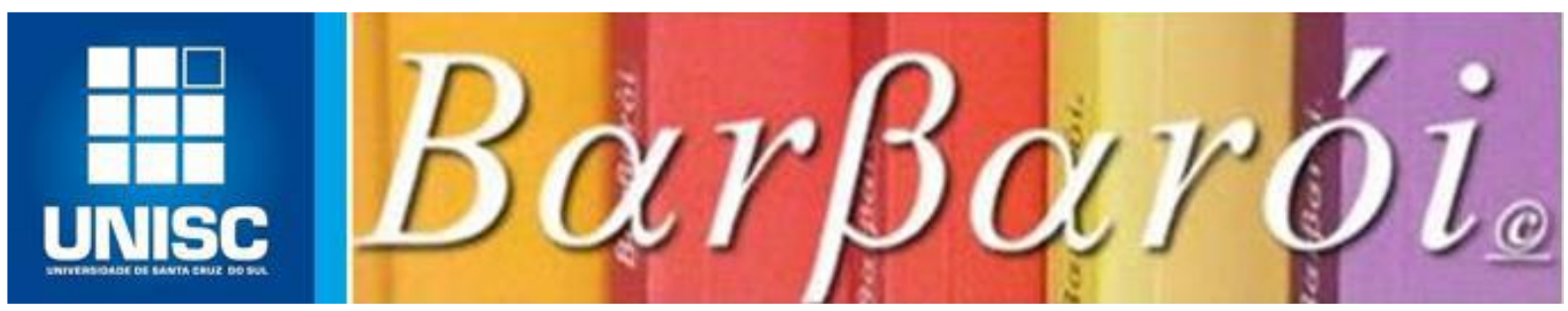

\title{
FATORES SOCIAIS QUE CONTRIBUEM PARA O ACOLHIMENTO INSTITUCIONAL DE IDOSOS
}

\author{
DOI: http://dx.doi.org/10.17058/barbaroi.v0i57.15212

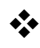 \\ Simone Vivian Moura \\ Universidade Federal de Jatai - UFJ - Brasil \\ $*$
}

\begin{abstract}
Resumo
Verifica-se um aumento significativo no contingente de pessoas idosas no Brasil. O Instituto Brasileiro de Geografia e Estatística estima que até o ano 2030, o percentual de idosos deverá superar o de crianças e adolescentes em cerca de quatro milhões. Grande parte de idosos necessitam de cuidados em decorrência da redução da capacidade funcional, no entanto, nem todos conseguem ser assistidos pelos seus familiares, pois existem fatores sociais que impedem que esse suporte seja praticado no seio familiar, como por exemplo, a vulnerabilidade socioeconômica. O presente artigo trata-se de uma revisão bibliográfica que discorre sobre os critérios para o acolhimento de idosos nas Instituições de Longa Permanência sob a ótica de garantia de direitos. O percurso metodológico do estudo aqui proposto aconteceu por meio de pesquisa bibliográfica documental. Verificou-se que o acolhimento institucional no âmbito da política de assistência social, ocorre em regime de internato, sendo indicado aos idosos sem vínculo familiar e com algum grau de dependência, bem como aos idosos em situação de violação de direitos.
\end{abstract}

Palavras-chave: Idoso. Família. Vulnerabilidade Socioeconômica. Acolhimento Institucional

\section{Introdução}

O processo de envelhecimento é um fenômeno social, histórico, cultural, e político que deve ser compreendido em diferentes contextos de vida social e pessoal do indivíduo. A partir do século XX, verificou-se o aumento da expectativa de vida nos países mais desenvolvidos e industrializados, expandindo-se gradualmente para os países em desenvolvimento (DE ARAÚJO NUNES; MENEZES; ALCHIERI, 2010).

O envelhecimento populacional brasileiro é um fato demográfico recente. As transformações no perfil demográfico da população iniciaram na década de 1960 e desde então cresce de maneira acelerada. O modelo de sociedade que era majoritariamente rural e 
tradicional, com famílias numerosas e alto índice de mortalidade infantil, apresentou-se como sociedade urbana, com novos arranjos familiares, diminuição da taxa de mortalidade e também de fecundidade. Para Chaimowicz (1997), a transição demográfica pode ser justificada pela assistência médico-sanitária, ao acesso a vacinas e antibióticos e, posteriormente, a partir da década de 1940, pelo alargamento do desenvolvimento tecnológico no âmbito da medicina.

Conforme dados da Organização Mundial de Saúde (OMS), a previsão é de que, em 2025, o Brasil contará com 1,2 bilhões de pessoas com mais de 60 anos. Isto posto, o país ocupará o sexto lugar no total de idosos (DE ARAÚJO NUNES; MENEZES; ALCHIERI, 2010). Essas alterações no perfil demográfico da população exigem uma rápida intervenção do Estado por meio de políticas públicas. Atualmente, para Ramos (2003) um dos grandes desafios para o poder público, é assegurar os direitos dos idosos, principalmente aqueles inseridos em contextos de vulnerabilidade social, com baixa escolaridade e alta grau de dependência, que conforme esse autor, é a realidade da maioria dos idosos brasileiros.

Verifica-se o esforço das autoridades em responder às crescentes demandas inerentes à população idosa. O país foi um dos primeiros a promulgar dispositivos legais como a Política Nacional Idoso, a Política Nacional de Saúde da Pessoa Idosa e o Estatuto do Idoso, os quais visam garantir direitos desse público, agregando-os na condição de cidadãos. Tais instrumentos jurídicos estabelecem a partilha de responsabilidades entre a família, o Estado e a sociedade e, priorizam a manutenção do idoso em seus lares, em detrimento do atendimento asilar. Contudo, conforme assinala Ramos (2003), um número importante de famílias, principalmente as multigeracionais que coabita com idosos, que na visão de Ramos, frequentemente são as mais pobres, não conseguem cuidar do parente idoso sem o suporte de políticas públicas. Para esse autor, o Estado delega parte das suas responsabilidades para essas famílias, o que compromete ainda mais o contexto de vulnerabilidade social.

Para Perlini; Leite; Furini (2007), a procura pelo acolhimento institucional de idosos, modalidade prevista na política pública de assistência social, tem crescido nos últimos anos. Elas explicam que em função do processo acelerado de industrialização, e com a inserção do maior número possível de membros da família no mercado de trabalho, em especial a mulher, essa classe trabalhadora enfrenta dificuldades para prestar os cuidados aos parentes idosos, o que aumenta as chances da institucionalização.

A assistência à velhice deve acontecer, preferencialmente, em seus lares. Entretanto, em situações que nem a família, tampouco os idosos tenham condições socioeconômicas para garantir essa assistência, cabe ao Estado oferecer auxílio e recursos no sentido de evitar a 
separação dos membros. Dessa forma, torna-se imperativo o desenvolvimento de políticas públicas que promovam a prevenção do acolhimento institucional. A situação de vulnerabilidade social não pode ser o único motivo para ocorrer a institucionalização da pessoa idosa.

A primeira seção do estudo aqui proposto discorre sobre a transição demográfica, enfocando as características do envelhecimento populacional no Brasil. A segunda seção discute sobre o papel da família no envelhecimento e destaca como a situação socioeconômica pode ser um fator dificultador da manutenção do idoso em seu lar. A terceira e última seção contempla a finalidade das Instituições de Longa Permanência para Idosos sob a ótica da Política Pública de Assistência Social brasileira. Por fim, são tecidas algumas considerações acerca dos desafios para garantir o direito do idoso à convivência familiar.

\section{Envelhecimento populacional no Brasil}

A queda de mortalidade, principalmente nos países desenvolvidos, apresentou ao mundo o fenômeno de envelhecimento populacional. Graças ao avanço da medicina, tecnologia, urbanização, saneamento e elevação dos níveis de higiene, ocorridos no final da década de 1940 e início dos anos 1950, os indivíduos puderam viver mais e com maior qualidade de vida (MIRADA; MENDES; SILVA, 2016). Para a Organização das Nações Unidas (ONU), identifica-se como idoso, o indivíduo com 60 anos ou mais, nos países em desenvolvimento, e com 65 anos ou mais nos países desenvolvidos (BRASIL, 2010).

Na visão de Zanon; Moretto; Rodrigues (2013), o envelhecimento pode ser compreendido como um processo que é impactado por fatores sociais, biológicos, culturais, econômicos, dentre outros. Para esses autores, o envelhecimento populacional é inerente às modificações na estrutura etária da população em decorrência da transição demográfica que se inicia com a redução das taxas de mortalidade e de fecundidade, tendo como consequência, o aumento da expectativa de vida.

Nas últimas décadas, conforme destaca Closs; Schwanke (2012), foi possível acompanhar o crescente número da população idosa e uma diminuição relativa na proporção de crianças e jovens. Tal fenômeno ocorreu de forma acelerada nos países em desenvolvimento, como o Brasil, ao passo que, nos países desenvolvidos o processo de envelhecimento aconteceu de forma gradual.

Meireles, et al., (2007) assinalam que até meados do século XX a chance de os brasileiros morrerem em meio ao período produtivo era grande. No entanto, em virtude dos 
avanços da ciência, tecnologia, acesso aos serviços preventivos de saúde, tratamento de doenças infectocontagiosas e crônico-degenerativas, utilização do saneamento básico, bem como estímulo para a adoção de hábitos saudáveis, notou-se uma elevação da vida média da população, mostrando que é possível envelhecer também em um país em desenvolvimento, como o Brasil. Veras; Oliveira (2018) corroboram na discussão e salientam que a partir do século XX, vivenciar a velhice deixou de ser privilégio de países desenvolvidos. Ainda nessa linha de raciocínio, para Miranda, Mendes, Silva (2016), o aumento da expectativa de vida dos brasileiros, iniciada na década de 1960 é sustentado pelos avanços tecnológicos no âmbito da saúde, sendo possível oferecer aos indivíduos tratamentos preventivo e curativo de várias doenças.

No entendimento de Chaimowicz (1997), a diminuição da taxa de mortalidade no Brasil que deu início à transição demográfica, é justificada pela assistência médico-sanitária com acesso a vacinas e antibióticos e, posteriormente, a partir da década de 1940, pelo alargamento do desenvolvimento tecnológico no âmbito da medicina. Esse autor ainda salienta que a alteração na base demográfica da população no Brasil iniciou a partir da década de 1960 em decorrência de um progressivo declínio nas taxas de mortalidade, bem como das taxas de fecundidade. Para Veras; Oliveira (2018), o processo de envelhecimento inicia com a queda sustentada de fecundidade. Eles explicam que tal fenômeno contribuiu para que a pirâmide etária do Brasil sofresse importantes modificações.

Para Carvalho; Garcia (2003), o processo de envelhecimento de uma população não é inerente apenas aos indivíduos, tampouco a uma geração, ele está atrelado às mudanças na estrutura etária da população, aumentando relativamente o número de pessoas acima de uma idade definidora do início da velhice. Para esses autores, a velhice é resultado do declínio da fecundidade e não da mortalidade, como o senso comum comumente afirma.

A longevidade num país como o Brasil, marcado pelas desigualdades sociais, é uma conquista, cita Areosa (2015). Veras; Oliveira (2018), destacam que assistir o envelhecimento populacional é gratificante, mas desde que ele seja vivenciado com qualidade, pois nesse processo, existem fatores biopsicossociais que merecem grande atenção de toda a sociedade. Para Vecchia, et al., (2005), não basta o idoso ter uma sobrevida maior, ele tem que ter qualidade de vida. Na visão desses últimos autores, a qualidade de vida no envelhecimento está relacionada aos aspectos como autoestima, nível socioeconômico, interação familiar e comunitária, valores culturais, éticos e religiosos, estado de saúde psíquica e física, dentre outros. 
Para o grupo World Health Organization Quality of Life (Grupo de Qualidade de Vida da Organização Mundial da Saúde), a qualidade de vida é a percepção do indivíduo de sua posição na vida no contexto da cultura e sistema de valores nos quais ele vive e em relação aos seus objetivos, expectativas, padrões e preocupações (WHOQOL,1995). Nessa discussão, Dawalibi, et al., (2013), aponta que o grupo WHOQOL avaliam seis domínios no processo de envelhecimento sob a ótica de qualidade de vida: "físico, psicológico, nível de independência, relações sociais, meio-ambiente e espiritualidade (religião e crenças pessoais)".

Na concepção de Areosa (2015), paralelamente ao aumento da expectativa de vida, surgem novas demandas do processo de envelhecimento ligados às áreas de saúde, assistência social, previdência, habitacional, tecnológica, educacional, bem como os cuidados de longa duração que preferencialmente devem ser ofertados pelos familiares. Para tanto, Dawalibi, et al., (2013), alertam que o processo de envelhecimento humano, sendo esse um fenômeno emergente e irreversível, deve ser encarado não como finitude, mas como um período que requer acolhimento, acompanhamento e ações preventivas por meio de iniciativas dos setores público e privado, com a devida participação da família e sociedade, para que assim seja possível garantir efetivamente os direitos da população idosa.

\section{O papel da família no processo de envelhecimento}

A família é formada por um conjunto de pessoas que têm vínculo parental e que residem no mesmo lar, de acordo com Figueiredo; Moser (2013). Para essas autoras, comumente, a função interna da família é o cuidado e a proteção dos seus membros, além da função externa, que é a transmissão de valores e culturas. A família para Silva; Santos; Souza (2014), é considerada como fonte de apoio e cuidado entre membros que estão conectados por laços de consanguinidade, os quais podem ser compartilhados ou não no mesmo ambiente.

Figueiredo; Moser (2013), explicam que, no momento em que a família assume os cuidados de um membro idoso, ela encontra-se na fase madura, ou seja, essa fase é marcada por uma reconfiguração de papéis ligado à uma trajetória histórica que apresenta um sujeito sendo cuidado por novos integrantes no mesmo grupo familiar, na perspectiva multigeracional. Marques; Souza (2012) assinalam que a literatura tem discutido sobre as dificuldades que muitas famílias vivenciam para cuidarem dos seus idosos, principalmente aquelas de baixa renda. Um estudo realizado por essas últimas autoras, sobre os aspectos de desconexão e alienação no contexto de vida de idosos sob o ponto de vista da integridade familiar, apontou 
que o risco de idosos inseridos em contextos de pobreza não alcançarem a integridade familiar é grande, principalmente nos casos em que esses não estejam em boa condição de saúde.

O maior indicador para a institucionalização de idosos para Caldas (2003), é a falta de suporte familiar. Contudo, algumas pesquisas revelam que grande parte dos idosos institucionalizados tinham vínculo familiar antes do acolhimento acontecer, como demonstra a pesquisa de Santana et al., (2016), realizada em uma Instituição de Longa Permanência para idosos (ILPI) sem fins lucrativos, na cidade Simão Dias/SE. Identificou-se por meio da referida pesquisa, a prevalência de idosos analfabetos, sexo feminino, de cor preta ou parda e com vínculos familiares. Esses últimos autores argumentam que pelos indicadores sociais apresentados, é possível concluir que esses idosos eram precedentes de contextos sociais mais fragilizados.

Outra pesquisa realizada com intuito de analisar o perfil dos idosos institucionalizado foi feita por Pinheiro et al., (2016), em uma ILPI sem fins lucrativos na cidade de Natal/RN. Os resultados também apontaram a predominância de idosos com referência familiar, do sexo feminino, cor preta ou parda e analfabetos. Os resultados de ambas as pesquisas sinalizam que pode existir um status social demarcado nas ILPIs sem fins lucrativos.

No que concerne aos cuidados com a pessoa idosa, a Constituição Federal de 1988 disciplina que:

\footnotetext{
Art. 229. Os pais têm o dever de assistir, criar e educar os filhos menores, e os filhos maiores têm o dever de ajudar e amparar os pais na velhice, carência ou enfermidade; Art. 230. A família, a sociedade e o Estado têm o dever de amparar as pessoas idosas, assegurando sua participação na comunidade, defendendo sua dignidade e bem estar e garantindo-lhes o direito à vida

$\S 1^{\circ}$ os programas de amparo aos idosos serão executados preferencialmente em seus lares (BRASIL, 1988).
}

Observa-se que no artigo 230, a família é primeira responsável devendo prover meios que garantam a sociabilidade, a dignidade e o direito à vida do idoso. Entretanto, diante das dificuldades de ordem socioeconômica dessas famílias para desempenhar tais funções, nota-se uma tendência do aumento de transferência dessa responsabilidade para as ILPIs (RAMOS, 2013). O Estatuto do Idoso, por sua vez, também estabelece ser de responsabilidade da família o cuidado com os idosos. No art. $3^{\circ}$ desse Estatuto cita que:

É obrigação da família, da comunidade, da sociedade e do Poder Público assegurar ao idoso, com absoluta prioridade, a efetivação do direito à vida, à saúde, à alimentação, à educação, à cultura, ao esporte, ao lazer, ao trabalho, à cidadania, à liberdade, à dignidade, ao respeito e à convivência familiar e comunitária (BRASIL, 2003). 
$\mathrm{Na}$ discussão de Carvalho (2005), os arranjos familiares foram obrigados a se redesenharem nos moldes do capitalismo monopolista, centrando-se na força de trabalho para a sobrevivência em função das transformações oriundas do processo de industrialização, dos avanços tecnológicos e da inserção da mulher no mercado. Ainda para Carvalho, na percepção das políticas públicas, a família é considerada responsável pela proteção dos indivíduos da mesma forma que o Estado.

Do ponto de vista legal, é muito claro que a responsabilidade inicial para com os idosos é da família, argumentam Souza; Skubs, Brêtas (2007). Porém, não existem ações públicas que avaliem se as famílias compreendem o processo de envelhecimento e se estão preparadas para os cuidados na perspectiva intergeracional. Ainda no debate dessas autoras, é esclarecido que as famílias de baixa renda apresentam mais dificuldades de compreensão do processo de envelhecimento, em virtude da pouca escolaridade e falta de acesso às informações sobre os direitos socioassistenciais no âmbito da seguridade social.

O envelhecimento não significa doença, incapacidades ou dependência. Apesar disso, nas idades mais avançadas, nota-se a prevalência de doenças crônicas e incapacidades funcionais. Isso significa que a família em situação de vulnerabilidade socioeconômica terá mais dificuldade para adaptar a residência ao idoso, caso seja necessário, contar com o suporte de um cuidador particular, bem como custear medicamentos, insumos, equipamentos terapêuticos, que porventura falte no serviço de dispensação gratuita, e demais necessidades inerentes à pessoa idosa (CAMARGOS, 2014).

No Brasil existem poucos programas por parte do Estado que visam oferecer suporte às famílias que não possuem recursos financeiros para acolher de forma integral o membro idoso, assinala Camargos (2014). A autora cita de forma positiva em seu artigo, um programa intitulado Maior Cuidado, coordenado pela política de assistência social da prefeitura de Belo Horizonte e executado por meio dos equipamentos públicos na área da saúde e assistência social. O objetivo desse programa é evitar ou protelar a institucionalização de idosos semidependentes e dependentes que têm referência familiar, mas que vivenciam situação de vulnerabilidade socioeconômica. A longevidade está condicionada ao contato e suporte social com a família e comunidade. É fundamental para a adaptação do idoso, de acordo com Areosa, et al., (2012), a sensação de pertencimento aos entes queridos para melhor aceitação da sua condição.

Torna-se importante conhecer e analisar as causas que levam as famílias a procurarem pelo serviço de institucionalização de idosos, bem como os critérios estabelecidos para o 
acolhimento no campo da assistência social a fim de evitar que tal medida ocorra de forma precoce ou de maneira equivocada.

\section{Instituições de Longa Permanência para Idosos}

A instituição asilar é uma das modalidades de acolhimento ao idoso mais antiga no contexto mundial. Para Camarano; Kanso (2010) a origem das ILPIs está ligada às instituições asilares sustentadas pela igreja católica e burguesia, que tinham como finalidade amparar os idosos, bem como outros indivíduos em situação de abandono, como por exemplo, pessoas com deficiência, viúvas, mães solteiras, crianças e adolescentes. Veras; Oliveira (2018) sinalizam que no início do século XX, a segregação de pessoas idosas predominava por meio das práticas equivocadas de internações em asilos, as quais eram permeadas por aspectos sociais, políticos e econômicos e que não existiam políticas públicas de proteção e amparo aos idosos.

Para Veras; Oliveira (2018), a partir da Constituição Federal de 1988, a política assistencialista em curso recebe uma conotação de direito de cidadania. Os idosos passam a ter uma atenção diferenciada, os quais poderão contar com uma rede de seguridade social composta pela saúde, assistência social e previdência social. Para essas autoras, na década de 1980, a comunidade científica por meio de realização de seminários e congressos começou a sensibilizar os governos e a população sobre o processo de envelhecimento.

Em setembro de 1989, o Ministério da Saúde expediu a Portaria n. 810 que estabeleceu as Normas para Funcionamento de Casas de Repouso, Clínicas Geriátricas e outras Instituições destinadas ao atendimento de idosos. Pela Lei n. 8.080 de 1990, foi criada a Política Nacional de Saúde (PNS) que especificou sobre as condições para a promoção, proteção e recuperação da saúde e também sobre a organização e o funcionamento dos serviços. A partir da promulgação dessa última Lei, legislações setoriais nessa perspectiva foram sendo criadas, como por exemplo a Política Nacional de Saúde do Idoso (PNSI) que se concretizou graças à PNS (PINTO; SIMSON, 2012).

Em 1993, outro avanço que merece destaque é promulgação da Lei n. 8.742 que preconiza proteção aos idosos que não têm condição de autossustento por meio do Benefício de Prestação Continuada (BPC), no valor de um salário mínimo. Em 1994, pela Lei n. 8.842, estabeleceu-se a Política Nacional do Idoso (PNI), que definiu como pessoa idosa aquela com 60 anos ou mais e destacou a prioridade do convívio familiar em detrimento do atendimento asilar, dentre outros. Essa última Lei foi resultado de intensa discussão no país, com ampla participação de idosos ativos, gerontólogos e a sociedade civil, sendo apontada a necessidade 
de se compreender a velhice de maneira diferenciada nos aspectos físicos, sociais, econômicos e político. Para a PNI, a institucionalização deve ter um caráter de excepcionalidade e brevidade. Ela ainda enfatiza a necessidade de se criar alternativas comunitárias de assistência ao idoso, como por exemplo, Repúblicas, Casa Lar, Centro de Convivência, Centro Dia e Atendimento Domiciliar. No entanto, a PNI não define regras de funcionamento destas modalidades, tampouco estabelece suas diferenças (PINTO; SIMSON, 2012).

Em 2003 a Lei n. 10.741 criou o Estatuto do Idoso, o qual consolidou os direitos conferidos pelas diversas leis federais, estaduais e municipais, estruturando-os por tópicos, especificando os direitos, deveres, bem como as punições para os infratores. Com o Estatuto, houve ampliação dos direitos da pessoa idosa, que passa também a ser tutelada por uma lei própria. O Estatuto ratifica a priorização do atendimento do idoso no interior da sua família, em detrimento do cuidado em instituições asilares. Até o ano 2005, as instituições de acolhimento institucional ainda eram conhecidas como asilos e atuavam de maneira distintas, ou seja, não havia um regulamento padrão para que tais instituições pudessem seguir. Nota-se que a nomenclatura "Instituição de Longa Permanência para Idosos" não encontra previsão na Política Nacional do Idoso, nem no Estatuto do Idoso (PINTO; SIMSON, 2012).

Em 26 de setembro de 2005, por meio da Resolução da Diretoria Colegiada (RDC n. 283) da Agência Nacional de Vigilância Sanitária (ANVISA), foi aprovado o Regulamento Técnico que estabeleceu as normas para as instituições de acolhimento institucional para idosos. Os asilos passam a ser chamados como Instituições de Longa Permanência para idosos e deverão seguir as normas da referida RDC. Para Camaro; Kanso (2010), as ILPIs apresentam aspectos residenciais destinadas ao domicílio coletivo de pessoas com idade igual ou superior a 60 anos, "com a função de integrar a rede de assistência social à rede de assistência à saúde". Além da moradia, é previsto ainda para os idosos, a alimentação, vestuário, práticas religiosas e esportivas, lazer, cultura, educação, acesso aos serviços médicos, medicamentos e acompanhamento por uma equipe multidisciplinar.

Atualmente, o órgão responsável pela execução de políticas para as ILPIs é o Ministério da Cidadania. Quanto à fiscalização, é realizada pela ANVISA e suas agências locais. As ILPIs são consideradas para o Ministério da Saúde, unidades de saúde de baixa complexidade, pelo fato de não requererem procedimentos e equipamentos de alta complexidade. Já para a política de assistência social, as ILPIs são consideradas de alta complexidade (PINTO; SIMSON, 2012).

A Resolução $n^{\circ}$ 109, de 11 de novembro de 2009, que aprova a tipificação dos serviços socioassistenciais, estruturou os serviços por níveis de complexidade do Sistema Único de 
Assistência Social (SUAS), em proteção básica e proteção social especial de média e alta complexidade. A modalidade de acolhimento institucional para idosos está prevista no serviço de proteção especial de alta complexidade:

\begin{abstract}
Acolhimento para idosos (as) com 60 anos ou mais, de ambos os sexos, independentes e/ou com diversos graus de dependência. A natureza do acolhimento deverá ser provisória e, excepcionalmente, de longa permanência quando esgotadas todas as possibilidades de autosustento e convívio com os familiares. É previsto para idosos (as) que não dispõem de condições para permanecer com a família, com vivência de situações de violência e negligência, em situação de rua e de abandono, com vínculos familiares fragilizados ou rompidos (BRASIL, 2009).
\end{abstract}

Percebe-se que o acolhimento institucional no âmbito do Sistema Único de Assistência Social é indicado aos idosos sem referência familiar e que não conseguem viver de maneira independente na sociedade, bem como aos idosos com vínculos familiares frágeis ou rompidos. Para Fernandes; Soares (2012), embora as legislações reconheçam a importância da convivência familiar no processo de envelhecimento, o Estado ainda continua a delegar às famílias dos idosos, responsabilidades que caberia a ele desempenhar. As autoras sinalizam sobre a escassez de políticas de suporte às famílias cuidadoras de idosos semidependentes e dependentes.

\title{
Considerações finais
}

No decorrer da discussão do artigo em tela, foi possível compreender que o processo de envelhecimento exige da família, envolvimento, disponibilidade para os cuidados, além de recursos financeiros. Os laços afetivos não deixam de existir após um ente envelhecer. Todavia, muitas famílias que não contam com o auxílio de políticas públicas locais, mesmo almejando amparar o membro idoso, não conseguem praticar tal suporte de forma independente.

Políticas públicas que possam garantir a permanência de idosos em seus lares por mais tempo ou definitivamente, são extremamente importantes para a garantia de direitos dessa população que merece cuidados especiais nessa fase de vida. $\mathrm{O}$ acolhimento institucional deve acontecer quando forem esgotadas todas as possibilidades de manutenção do idoso em seu núcleo familiar. Além disso, acredita-se que são necessárias ações de iniciativas públicas que identifiquem e monitorem as famílias com dificuldades em oferecer cuidados aos idosos, para que dessa maneira seja possível viabilizar mecanismos de suporte e cuidado em domicílio. 


\title{
SOCIAL FACTORS THAT CONTRIBUTE TO THE INSTITUTIONAL CARE OF ELDERLY
}

\begin{abstract}
There is a significant increase in the number of elderly people in Brazil. The Brazilian Institute of Geography and Statistics estimates that by the year 2030, the percentage of elderly people should exceed that of children and adolescents by around four million. Most elderly people need care due to reduced functional capacity, however, not all of them are able to be assisted by their families, as there are social factors that prevent this support from being practiced within the family, such as socioeconomic vulnerability. The purpose of this article is to conduct a discussion on the criteria for the reception in Long Term Care Institutions for the Elderly from the perspective of guaranteeing rights. The methodological path of the study proposed here took place through documentary bibliographic research. It was found that institutional care within the scope of the social assistance policy occurs in a boarding school, being indicated to the elderly without family ties and with some degree of dependence, as well as to the elderly in situations of violation of rights.
\end{abstract}

Keywords: Elderly. Family. Socioeconomic Vulnerability. Institutional Reception.

\section{FACTORES SOCIALES QUE CONTRIBUYEN AL CUIDADO INSTITUCIONAL DE LOS ANCIANOS}

\section{Resumen}

Hay un aumento significativo en el número de personas mayores en Brasil. El Instituto Brasileño de Geografía y Estadística estima que para el año 2030, el porcentaje de personas mayores debería superar el de niños y adolescentes en alrededor de cuatro millones. La mayoría de las personas mayores necesitan atención debido a la capacidad funcional reducida, sin embargo, no todas pueden recibir asistencia de sus familias, ya que existen factores sociales que impiden que este apoyo se practique dentro de la familia, como la vulnerabilidad socioeconómica. El propósito de este artículo es llevar a cabo una discusión sobre los criterios para la recepción en instituciones de atención a largo plazo para personas mayores desde la perspectiva de garantizar los derechos. La ruta metodológica del estudio propuesto aquí tuvo lugar a través de la investigación bibliográfica documental. Se encontró que la atención institucional dentro del alcance de la política de asistencia social ocurre en un internado, siendo indicada a los ancianos sin vínculos familiares y con cierto grado de dependencia, así como a los ancianos en situaciones de violación de derechos.

Palabras clave: Ancianos. Familia. Vulnerabilidad Socioeconómica. Recepción Institucional.

\section{REFERÊNCIAS}

AREOSA, S. V. C.; Idosos Provedores: a importância dos recursos da aposentadoria para as famílias brasileiras. Barbarói, Santa Cruz, v. 1, n. 45, p. 173-184, jul./dez., 2015.

AREOSA, S. V. C.; ARAÚJO, C. K., CARDOSO, C. M. C., MOREIRA, E. P. M. Envelhecimento: relações pessoais e familiares. Barbarói, Santa Cruz, v.36, ed. esp., p.120132, jan./jun. 2012.

BRASIL. Ministério da Saúde. Atenção à saúde da pessoa idosa e envelhecimento, Brasília: Ministério da Saúde, 2010. 
Resolução da Diretoria Colegiada - RDC n⿳ 283, de 26 de setembro de 2005. Aprova o Regulamento Técnico para o Funcionamento das Instituições de Longa Permanência para Idoso.

. Resolução ${ }^{\circ} 109$ de 11 de novembro de 2009. Aprova a Tipificação Nacional de Serviços Socioassistenciais.

Constituição da República Federativa do Brasil, de 5 de outubro de 1988.

CALDAS, Célia Pereira. Envelhecimento com dependência: responsabilidades e demandas da família. Cadernos de Saúde Pública, v. 19, p. 733-781, 2003.

CAMARANO, Ana Amélia; KANSO, Solange. As instituições de longa permanência para idosos no Brasil. Rev. bras. estud. popul., São Paulo, v. 27, n. 1, p. 232-235, June 2010. Available from http://www.scielo.br/scielo.php?script=sci_arttext\&pid=S0102$30982010000100014 \& \operatorname{lng}=\mathrm{en} \& \mathrm{nrm}=\mathrm{iso}>$.access on 20 May 2020. https://doi.org/10.1590/S0102-30982010000100014.

CAMARGOS, Mirela Castro Santos. Instituições de longa permanência para idosos: um estudo sobre a necessidade de vagas. Revista Brasileira de Estudos de População, v. 31, n. 1, p. 211-217, 2014.

CARVALHO, José Alberto Magno de; GARCIA, Ricardo Alexandrino. O envelhecimento da população brasileira: um enfoque demográfico. Cad. Saúde Pública, Rio de Janeiro, v. 19, n. 3, p. 725-733, June 2003. Available from

<http://www.scielo.br/scielo.php?script=sci_arttext\&pid=S0102-

311 X2003000300005\&lng=en\&nrm=iso >.access

on 19 May 2020. https://doi.org/10.1590/S0102-311X2003000300005.

CARVALHO, Maria do Carmo Brant de. O lugar da família na política social. In: A família contemporânea em debate. 6. ed. São Paulo: Cortez, 2005, p. 15-22.

CHAIMOWICZ, Flávio. A saúde dos idosos brasileiros às vésperas do século XXI:

problemas, projeções e alternativas. Revista de saúde pública, v. 31, p. 184-200, 1997.

CLOSS, Vera Elizabeth; SCHWANKE, Carla Helena Augustin. A evolução do índice de envelhecimento no Brasil, nas suas regiões e unidades federativas no período de 1970 a 2010. Revista brasileira de geriatria e gerontologia, v. 15, n. 3, p. 443-458, 2012.

DAWALIBI, Nathaly Wehbe et al. Envelhecimento e qualidade de vida: análise da produção científica da SciELO. Estudos de Psicologia (Campinas), v. 30, n. 3, p. 393-403, 2013.

DE ARAÚJO NUNES, Vilani Medeiros; DE MENEZES, Rejane Maria Paiva; ALCHIERI, João Carlos. Avaliação da qualidade de vida em idosos institucionalizados no município de Natal, Estado do Rio Grande do Norte. Acta Scientiarum. Health Sciences, v. 32, n. 2, p. 119-126, 2010. 
FERNANDES, Maria Teresinha de Oliveira; SOARES, Sônia Maria. O desenvolvimento de políticas públicas de atenção ao idoso no Brasil. Revista da Escola de Enfermagem da USP, v. 46, n. 6, p. 1494-1502, 2012.

FIGUEIREDO, Tatiana Enter; MOSER, Liliane. Envelhecimento e Família: reflexões sobre a responsabilização familiar, os desafios às políticas sociais e a regulamentação da profissão de cuidador de pessoa idosa. In: Anais do Congresso Catarinense de Assistentes Sociais.

2013.

MARQUES, Filipa D.; SOUSA, Liliana Xavier Marques de. Integridade familiar e o idoso pobre: valores e significados*. Psicol inf., São Paulo, v. 16, n. 16, p. 11-

43, dez. 2012.Disponível em

$<$ http://pepsic.bvsalud.org/scielo.php?script=sci_arttext\&pid=S1415-

$88092012000200002 \& \operatorname{lng}=$ pt\&nrm=iso> . acessos em 19 maio 2020.

MEIRELES, Viviani Camboin et al. Características dos idosos em área de abrangência do Programa Saúde da Família na região noroeste do Paraná: contribuições para a gestão do cuidado em enfermagem. Saúde e Sociedade, v. 16, n. 1, p. 69-80, 2007.

MIRANDA, Gabriella Morais Duarte; MENDES, Antonio da Cruz Gouveia; SILVA, Ana Lucia Andrade da. O envelhecimento populacional brasileiro: desafios e consequências sociais atuais e futuras. Revista Brasileira de Geriatria e Gerontologia, v. 19, n. 3, p. 507 $519,2016$.

ORGANIZAÇÃO MUNDIAL DA SAÚDE. Envelhecimento ativo: uma política de saúde. Organização Pan-Americana da Saúde-OPAS, 2005.

PERLINI, Nara Marilene O. Girardon; LEITE, Marinês Tambara; FURINI, Ana Carolina. Em busca de uma instituição para a pessoa idosa morar: motivos apontados por familiares.

Revista da Escola de Enfermagem da USP, v. 41, n. 2, p. 229-236, 2007.

PINHEIRO, N.C. G; HOLANDA, V.C.D; MELO, L. A.; MEDEIROS, A. K. B.; LIMA, K. C. Desigualdade no perfil dos idosos institucionalizados na cidade de Natal, Brasil. Ciênc Saúde Coletiva. 2016;21(11):3399-3405.

PINTO, Silvia Patricia Lima de Castro; SIMSON, Olga Rodrigues de Moraes Von. Instituições de longa permanência para idosos no Brasil: sumário da legislação. Revista Brasileira de Geriatria e Gerontologia, v. 15, n. 1, p. 169-174, 2012.

RAMOS, Luiz Roberto. Fatores determinantes do envelhecimento saudável em idosos residentes em centro urbano: Projeto Epidoso, São Paulo. Cad. Saúde Pública, Rio de Janeiro, v. 19, n. 3, p. 793-797, June 2003. Available from <http://www.scielo.br/scielo.php?script=sci_arttext\&pid=S0102$311 X 2003000300011 \& \operatorname{lng}=$ en $\& n r m=$ iso $>$. access on 21 May 2020. https://doi.org/10.1590/S0102-311X2003000300011.

SANTANA, J. D. de M. et al. Participação dos familiares na vida dos idosos institucionalizados no lar São Francisco de Assis em Simão Dias/SE. UNISEPE Mantenedora

- Revistas Eletrônicas - Saúde em Foco, n. 08, 2016. Disponível em: < 
http://unifia.edu.br/revista_eletronica/revistas/saude_foco/saude.html\#>. Acesso em: 19 mai. 2020 .

SILVA, K. M. SANTOS, S. M. A. SOUZA, A. I. K. Reflexões sobre a necessidade do cuidado humanizado ao idoso e família. Saúde Transform Soc. 2014;5(3):20-4.

SOUZA, Rosangela Ferreira de; SKUBS, Thais; BRÊTAS, Ana Cristina Passarella. Envelhecimento e família: uma nova perspectiva para o cuidado de enfermagem. Revista Brasileira de Enfermagem, v. 60, n. 3, p. 263-267, 2007.

VECCHIA, Roberta Dalla et al. Qualidade de vida na terceira idade: um conceito subjetivo. Revista brasileira de epidemiologia, v. 8, n. 3, p. 246-252, 2005.

VERAS, Renato Peixoto; OLIVEIRA, Martha. Envelhecer no Brasil: a construção de um modelo de cuidado. Ciência \& Saúde Coletiva, v. 23, p. 1929-1936, 2018.

WHOQOL Group 1995. The World Health Organization Quality of Life Assessment (WHOQOL): position paper from the World Health Organization. Social Science and Medicine 41(10):1.403-1.409.

ZANON, R. R.; MORETTO, A. C.; RODRIGUES, R. L. Envelhecimento populacional e mudanças no padrão de consumo e na estrutura produtiva brasileira. Revista Brasileira de Estudos de População, v. 30, p. S45-S67, 30 dez. 2013.

Data de recebimento: $26 / 05 / 2020$

Data de aceite: 05/08/2020

\section{Sobre a autora:}

Simone Vivian Moura possui graduação em Serviço Social pela Faculdade de Ciências Econômicas, Administrativas e Contábeis de Divinópolis (2009); especialização em Instrumentalidade do Serviço Social pela Universidade de Viçosa (2013); mestranda do curso Gestão Social, Educação e Desenvolvimento Local pelo Centro Universitário UNA. Atualmente é tutora do curso superior em Serviço Social da Faculdade Pitágoras (desde 2019); Assistente Social da Polícia Militar do Estado de Minas Gerais (desde 2018) e Assistente Social da Associação Mineira de Reabilitação (desde 2009). Endereço Eletrônico: simonevivianmoura@hotmail.com 